\title{
ALL OPTICAL 3R REGENERATION AND WAVELENGTH CONVERTION
}

\author{
Davide M. Forin, Franco Curti, Giorgio M. Tosi-Beleffi, Francesco Matera ${ }^{1}$, \\ Andrea Reale, Silvello Betti, Simone Monterosso, Alessandro Fiorelli ${ }^{2}$, \\ Michele Guglielmucci, Sergio Cascelli ${ }^{3}$ \\ ${ }^{I}$ Ugo Bordoni Foundation, Via Baldassarre Castglione. No. 59,00142Rome, Italy; \\ Tel: (+39)0654802235,Fax: (+39)0654804402,e-mail:dforin@fub.it \\ ${ }^{2}$ Department of Electronic Engineering, University of Tor Vergata, Via del Politecnico 1, \\ 00133 Rome, Italy \\ ${ }^{3}$ Istituto Superiore delle Comunicazioni e Tecnologie dell'Informazione, Viale America \\ 201, Rome, Italy
}

Abstract: We experimentally demonstrate the possibility to realize an all-optical $3 R$ regenerator wavelength converter, based on a three-stage cascade configuration. A multi-wave-mixing based on the phase modulation of an auxiliary optical carrier induced by a two-signals interaction is used to perform simultaneously reshaping, re-amplification and wavelength conversion of a propagated signal. A fiber ring based unit is used to perform the all-optical timing extraction while an orthodox four-wave-mixing process inside a dispersion-shifted fiber is adopted for the retiming process. The work is patent pending.

\section{INTRODUCTION}

Optical devices and subsystems like wavelength converter for optical cross connects and 2R-3R optical regenerators are under development because of their fundamental role in the future optical communication systems.

3R regeneration preserves data quality and allows for improved transmission distances, thus enhancing transparency, scalability, and flexibility of optical networks. In this work we present results concerning the three main cascaded 
function to obtain a $3 \mathrm{R}$ regenerator: re-amplification and reshaping, timing extraction and re-sampling.

The Re-shaping and the Re-amplification unit (2RU) is studied via the simulations and the experimental results, at $10 \mathrm{Gbit} / \mathrm{s}$, regarding a new technique (patent pending) based on the adoption of an auxiliary continuous-wave incoming optical carrier. The characteristic non-linearity of a particular dispersion shifted optical fiber has been Exploited [1]. In that cases the envelope of the beating between a strong pump and a modulated signal, near the zero dispersion area (around $1539 \mathrm{~nm}$ ), determined a phase modulation of the whole incoming optical field; this beating generate a modulation of the refractive index of the fiber with frequency equal to frequency difference between pump and signal. Then any other carrier in the fiber is phase modulated. Hence the phase-modulated new carrier's spectrum presents a series of replicas typical of the phase modulation, each one reproducing the high data rate amplitude modulation of the original incoming signal enhancing, in this manner, the overall device wavelength conversion range. Furthermore, the in-out power-transfer characteristic of the n-th order replica is alike the correspondent order Bessel function, a reshaping function occurs too.

The Clock Recovery unit (CRU) is based on a fiber ring laser, which contains a single semiconductor optical amplifier (SOA), as active device, which provides both gain and modulation. The ring circuit was tested with both pseudorandom data sequences and periodic data patterns up to $10 \mathrm{~Gb} / \mathrm{s}$ generating clock pulse trains across a $30 \mathrm{~nm}$ tuning range. The operation of the present clock recovery ring based structure relies on the fast gain saturation of the SOA, induced by the incoming data stream, that generates the modulation in the cavity and determines the injection locking of the fiber laser.

The standard mode-locking SOA ring based technique has been extended introducing a Fabry-Perot cavity to select the right oscillating frequency [4]. Preliminary results, not reported here, shown that this configuration is able to works at $40 \mathrm{Gbit} / \mathrm{s}$ too. Desirable features of the CRU unit are the wide repetition frequency and wavelength locking ranges and the broad wavelength tuning range of recovered signal that it may provide.

The Re-Sampling unit (RU), is based on the usual FWM non-linear effect. The incoming modulated signal, in fact, is coupled with the pulsed optical clock signal, recovered by the second stage, and injected into a $6 \mathrm{~km}$ long standard dispersion shifted fiber (DS). The effect is a re-sampled optical replica of the input modulated signal.

In order to improve the performances of the non-linear effect we used a clock signal obtained by cross-gain modulation effect (XGM) into a semiconductor optical amplifier (SOA) in order to narrow the optical spectrum of the clock. 


\section{DESCRIPTION OF THE THREE STAGE SET-UP}

The key element of the reshaping section is a $10 \mathrm{~km}$ long DS fiber, with loss $=0.2 \mathrm{~dB} / \mathrm{km}$, nonlinear coefficient $=2.2\left[\mathrm{~W}^{*} \mathrm{~km}\right]-1$, chromatic dispersion $\mathrm{D}=0.19$ $\overline{\mathrm{ps}} / \mathrm{nmKm}$ (at the pump wavelength $1542.14 \mathrm{~nm}$ ), dispersion slope $\mathrm{SO}=0.0634$ $\mathrm{ps} / \mathrm{nm} 2 \mathrm{~km}$ and differential group delay (PMD factor) $\mathrm{DGD}=0.13 \mathrm{ps}[2]$.

Two DFB (DFB Source and DFB pump) laser sources used to generate respectively a signal (S), at $\lambda=1542.9 \mathrm{~nm}$, and a cw pump (CWP1), at $\lambda=1542.1$ $\mathrm{nm}$, are mixed together, in order to induce the requested non-linearity, as reported in Fig. 1 .

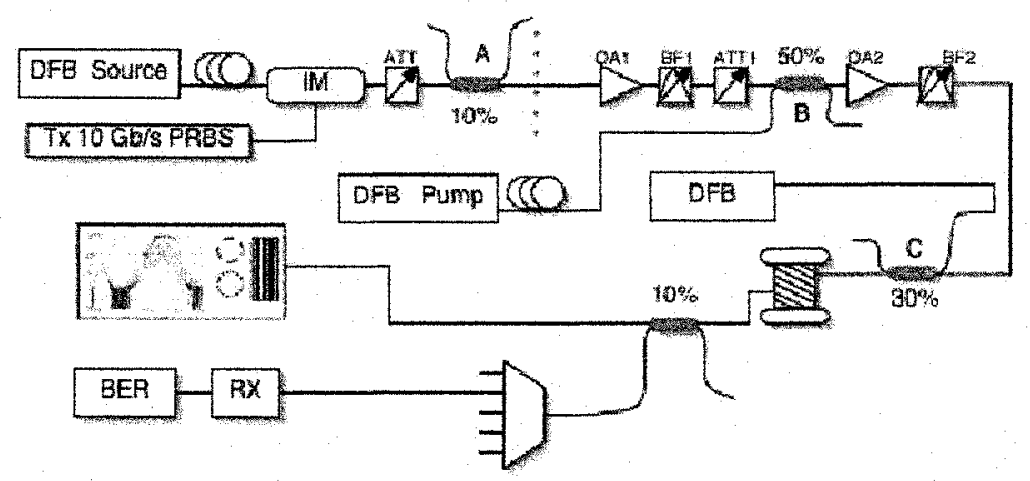

Figure 1. 2R unit set- up

In order to restrict the power depletion, due to Brillouin scattering phenomena,

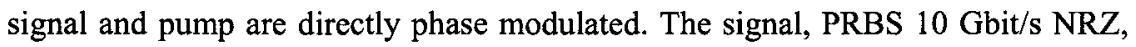
passes through a $10 \%$ coupler (A), preceded by an attenuator (ATT), used to measure the real optical input at the entrance of wavelength converter reshaper (WCR). After the 10\% coupler there is a block made up of an EDFA (OA1) and a band pass filter (BF1) followed by an attenuator (ATT1) that allow us to set the value of signal power for optimum reshaping function. Combination of signal and pump, through $50 \%$ (B)coupler, is processed by a block made up of an erbiumdoped power amplifier (OA2) and a band pass filter (BF2). This block increases the power of the pump and the signal up to the power needed in the non-linear processes. 


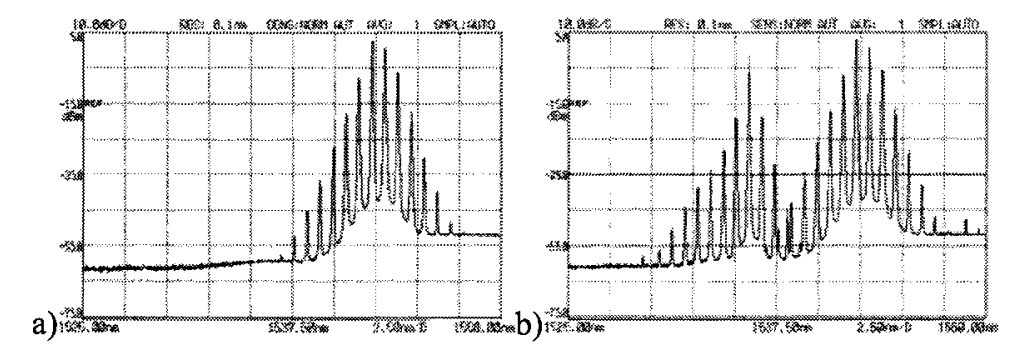

Figure 2. a)spectrum at the DS output of the beating between a cow pump and a modulated signal, b)spectrum at the DS output of the sum of beating and the third carrier

A third DFB (DFB) laser source is used to inject, through a $30 \%$ coupler (C), the auxiliary continuous-wave optical carrier (CW2) that, modulated along the propagation by the non-linearity, will present at the DS output a comb-like optical spectrum. The overall optical field spectrum has been reported in Fig. $2 b$.

Fig. 3 shows the experimental layout related to the Timing Extraction Stage.

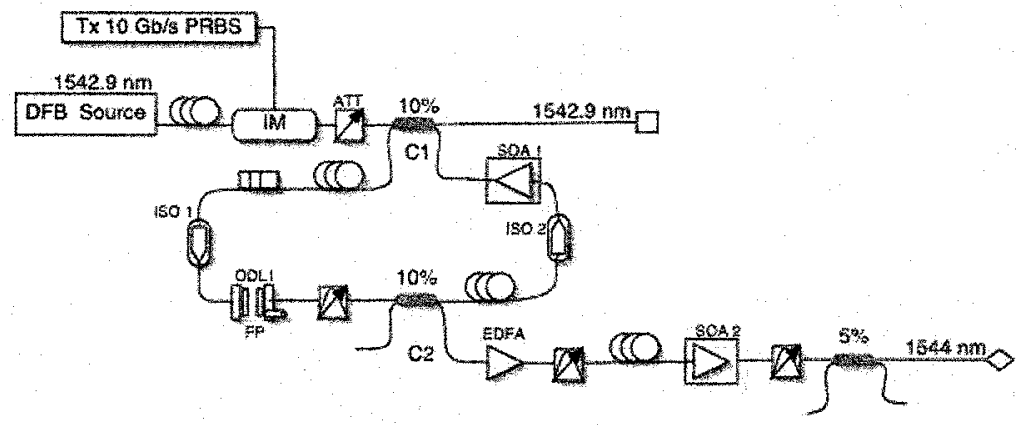

Figure 3. Clock Recovery Unit set-up

The test data patterns were produced modulating a DFB laser source which operates at $1542.9 \mathrm{~nm}$, with a lithium niobate external modulator driven by a 10 Gbit/s PRBS pattern generator. This incoming non-return-to-zero (NRZ) data pattern, injected into the loop circuit with a $10 \%$ optical fiber coupler $(\mathrm{C} 1)$, goes into an Alcatel M128 bulk model SOA1, with a $0.5 \mathrm{~mm}$ cavity length, $20 \mathrm{~dB}$ gain for small signal and a $40 \mathrm{~nm}$ amplification bandwidth, and is blocked by a first faraday isolator (ISO 1).

A second faraday isolator (ISO2), was inserted into the ring to ensure unidirectional counter clock-wise oscillation. Starting from the ISO2, the oscillation goes into a first stage, made by a polarizer followed by a polarization controller, able to optimize the oscillating polarization state for the SOAl. This 
stage is followed by ISO2 and by a second block made by a Fabry-Perot cavity (FP), realized with two optical mirrors whose reflectivity is equal to $99 \%$, and a variable optical delay line (ODL1). A subsequent $1 \mathrm{~nm}$ optical tunable filter is used to select the wavelength needed. The $-30 \mathrm{dBm}$ clock signal, extracted via a $10 \%$ coupler (C2), goes into an EDFA, a $1 \mathrm{~nm}$ bandwidth optical filter able to erase the ASE contribution and then is subsequently processed by an external SOA (SOA2), an Opto-Speed 1550MRI/X model with $30 \mathrm{~dB}$ gain for small signal and $30 \mathrm{~nm}$ amplification bandwidth, to obtain amplification and to simultaneously erase the amplitude noise. This particular configuration permit to obtain a sensible enhancement of the pulses train extracted.

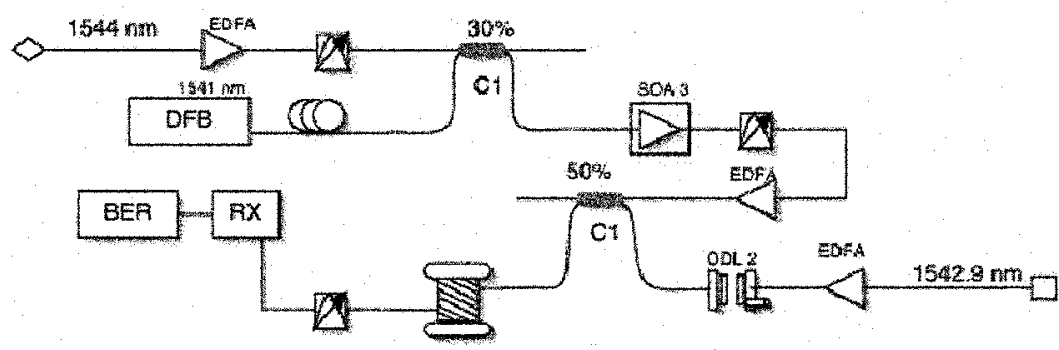

Figure 4. Re-Timing Unit set-up

Fig. 4 shows the experimental layout related to the Re-Sampling Stage. After $\mathrm{SOA} 2$, the recovered clock information at $1544 \mathrm{~nm}$, is injected into a SOA (SOA3), an Alcatel M18 bulk model, with a CW signal through a $30 \%$ coupler. The cross gain modulation inside the SOA3 modulates the $\mathrm{CW}$ pump with the same information carried by the signal coming from the ring. In this manner we have a clock information with a narrower optical spectrum. At this point this signal at $1541 \mathrm{~nm}$ is coupled with the original $10 \mathrm{Gbit} / \mathrm{s}$ NRZ PRBS signal at $1542.9 \mathrm{~nm}$, through a $50 \%$ coupler, and amplified in order to perform the retiming process inside a $6 \mathrm{~km}$ long DS fiber via a FWM process. An OPL2 is used to set the exact position of the data pulses in order to match it with the ones coming from the clock.

\section{DISCUSSION OF THE RESULTS}

To explain the generation of a so high number of components, we could argue that the non linear optical Kerr effect determines a phase modulation due to the beating between the pump and the signal to be converted, near $\lambda_{0}(1539 \mathrm{~nm}$ in our case); this beating cause a modulation of the refractive index of the fiber with 
frequency equal to frequency difference between pump and signal. This effect produce a phase modulation of the whole incoming field that generates new frequencies spaced by the pump-to-signal detuning range $\left(\omega_{\mathrm{p}}-\omega_{\mathrm{S}}\right)$ [3]. Then any other carrier present in the fiber is also phase modulated, and the phase-modulated carrier's spectrum presents a series of replicas typical of the phase modulation. The transfer function of these channels shows a non-zero threshold and a spread maximum that we can exploit for reshaper use. By adjusting the signal and pump power, at the DS fiber input, it is possible to choose the better working point in order to reshape the incoming corrupted signal, as reported in fig. 5.
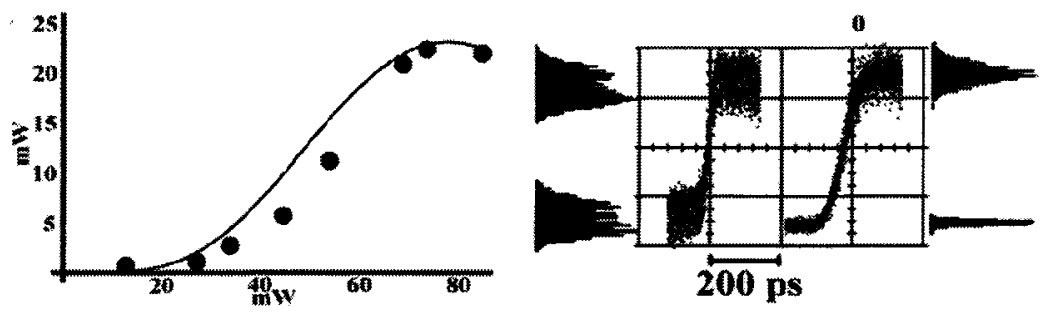

Figure 5. Comparison between experimental transfer function, dotted line, of $\mathrm{Ch} .47$ with simulation, continuous-line. Noise compression in a Non-Return-to-Zero $10 \mathrm{Gbit} / \mathrm{s}$ coded signal

The behavior of the converted signals depends both on the SNR, due to the different generation efficiency, and to the chromatic dispersion regime seen by each channel along the device. The non-linearity at the base of the process is able produce a phase modulation of the whole incoming field not only the optical beating field, that induce the non-linearity itself, but any kind of other optical carrier propagating with the beating field the fiber. Subsequently, the optical spectrum of the new carrier, at $1535.8 \mathrm{~nm}$ in this case, will present a series of components each one reproducing the high data rate amplitude modulation of the original incoming signal and each one with the same Bessel-like transfer function.

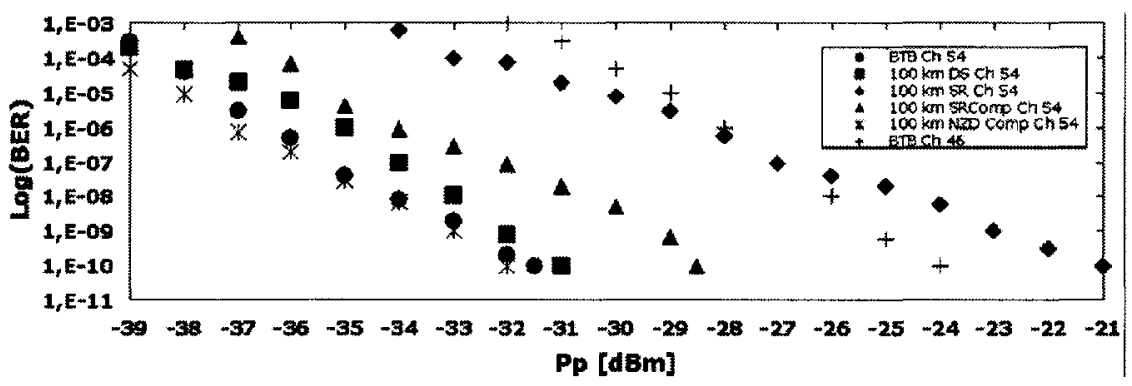

Figure 6. BTB and propagated BER measurements vs received power for ITU $\mathrm{Ch} 46$ and 54 
In Fig. 6 we report the BER results obtained at $10 \mathrm{Gbit} / \mathrm{s}$, in the back-to-back configuration and in propagation, for the ITU-GRID channels $54(1534.2 \mathrm{~nm})$ and $46(1540.5 \mathrm{~nm})$. The propagation was performed using a cable deployed between the cities of Rome and Pomezia encompassing different kind of fibers, G.652, G.653 and G.655. The better performance on the Ch.54 depends on the better signal to noise ratio respect to the Ch. 46 .

In Fig. 7a we report the clock information at the output of the SOA2 used to obtain amplification and to simultaneously erase the amplitude noise from the ring.

Regarding the CRU unit and in the case of NRZ signals, the Fabry-Perot cavity plays a key role since it simultaneously filters out a big amount of modes, that oscillate inside the cavity, and, furthermore, by tuning the mirrors with a piezodriver control, forces the cavity to resonate at the repletion incoming data rate. Without such resonance the locking conditions, in the case of NRZ signals, can be extremely difficult. The reasons of such behavior stand on the large frequency band of resonance of the ring. If the FP is instead introduced in the loop and its band is tuned around the frequency of the data rate input, it will select the exact oscillating frequency giving raise to a pulse trains. The cavity fundamental frequency was $7.5 \mathrm{MHz}$ and the locking bandwidth of the ring has been measured and it is equal to about $5 \mathrm{MHz}$.
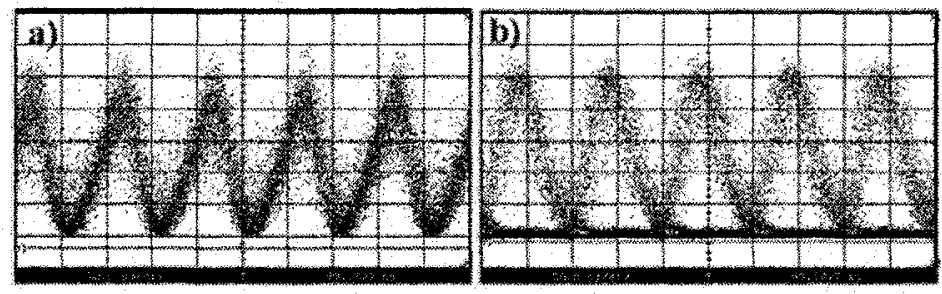

Figure 7. a) Clock recovered at the output of SOA2. b) Retiming of the incoming signal

In Fig. $7 \mathrm{~b}$ we report a preliminary result concerning the converted replica at $1544.7 \mathrm{~nm}$ obtained at the end of the $6 \mathrm{~km}$ long DS fiber. The FWM is obtained by the beating of the incoming signal at 1542.9 with the clock extracted at the output of the SOA2 at $1541 \mathrm{~nm}$. It is possible to see that the retiming process, due to width of the generates clock pulses, determines a format translation from NRZ to RZ format.

\section{CONCLUSIONS}

We have investigated three different stages able to implement the following functions: $2 \mathrm{R}$ regeneration and wavelength conversion, timing extraction and 
signal re-sampling. The results demonstrate the possibility to adopt a common cascade configuration in order to implement an all-optical $3 \mathrm{R}$ regeneration device.

\section{ACKNOWLEDGMENTS}

This work has been carried out in the framework of the Italian project FIRB RBAU01XEEM_005 "Photonics Networks" and in the cooperation between Fondazione Ugo Bordoni and Ministero delle Comunicazione under the project INTERN.IT

We thank ISCTI (Istituto Superiore delle Comunicazioni e Tecnologie dell'Informazione of Rome) supplying of instrumentation and the Rome-Pomezia experimental deployed cable.

\section{REFERENCES}

[1] F.Curti, F. Matera and Giorgio Maria Tosi-Beleffi, "Wavelength converter-reshaper based on multi-wavelength spectral components generation in optical fiber", Opt Comm, 208, pp. 85-89, 2002.

[2] M. Karlsson, Four-wave mixing in fiber with randomly varying zero-dispersion wavelength, J. Opt. Soc. Am. B, vol. 15, pp. 2269-2275, 1998.

[3] R. Thompson, R. Roy, "Nonlinear dynamics of multiple four-wave mixing processes in a single-mode fiber", Physical Review A, vol. 43, No. 9, pp. 4987-4996, 1991.

[4] J.P. Turkiewicz, E. Tangdiongga, All Optical $10 \mathrm{Ghz}$ Clock Recovery from $160 \mathrm{Gbit} / \mathrm{s}$ OTDM signals using a mode-locked fiber ring laser, IEEE/LEOS Benelux Chapter 2002, Amsterdam 